\title{
Thermopile effect due to laser radiation heating in thin films of high- $\mathrm{T}_{c}$ materials
}

\author{
K.F. Renk, J. Betz, S. Zeuner, H. Lengfellner, and W. Prettl
}

Institut für Angewandte Physik, Universität Regensburg, D-93040 Regensburg, Germany

We report on the observation of a thermopile effect caused by laser radiation heating in thin films of high- $T_{c}$ material. The thermopile effect is due to a transverse Seebeck effect. The transverse Seebeck effect is observable first because of the anisotropy of $\mathrm{YBa}_{2} \mathrm{Cu}_{3} \mathrm{O}_{7-\delta}$, and secondly because it is possible to grow off-axis epitaxial films. The thermopile effect can be used for developing detectors for laser radiation.

\section{Introduction}

Various groups have observed voltaic signals of laser irradiated $\mathrm{YBa}_{2} \mathrm{Cu}_{3} \mathrm{O}_{7-\delta}(\mathrm{YBCO})$ films $[1-4]$. There were speculations about the origin. It was discussed that a photoelectric [5] or a piezoelectric or a pyroelectric [6] effect may play a role. Recently, it was demonstrated that the main effect of the voltaic signal is due to a transverse Seebeck effect $[4,7,8]$. In YBCO films one can observe, to our knowledge for the first time, this thermovoltaic effect.

\section{The transverse Seebeck effect}

The transverse Seebeck effect can be observed for a YBCO film on strontium titanate, that has the c-axis not perpendicular to the surface of the film but with the c-axis tilted, i.e. the ab-plane is not parallel to the film surface, but tilted (Fig. 1). Such films can be grown by laser ablation using a substrate that is cut in an appropriate way. Irradiation of a film at room temperature with a laser pulse can result in a signal that can reach values up to $200 \mathrm{~V}$ (Fig. 1). The origin is a transverse Seebeck effect. While laser irradiation results in a temperature gradient perpendicular to the film surface a thermoelectric voltage is observed across the film the thermoelectric voltage is perpendicular to the temperature gradient.

Laser radiation is absorbed in the upper part of the film and creates a temperature gradient within the film. The heat in the film escapes into the substrate within a time of about $200 \mathrm{~ns}$. One may describe the device as an atomic layer thermopile because copper oxide layers and the interlayers between the copper oxide layers represent atomic thermoelements connected in series. The area is $10 \mathrm{~mm} \times 10 \mathrm{~mm}$ and the thickness of the film about $3000 \AA$.

If one reverses the temperature gradient by shining light not from the free film surface but from the side of the (transparent) substrate side, then the signal is reversed.

Instead of a laser beam we have also used a thermal source and could also observe thermoelectric signals $[4,7]$.

Usually one deals with the longitudinal thermoelectric effect. If we apply a temperature gradient along the $\mathrm{c}$-axis of an YBCO crystal then we find a thermoelectric field 


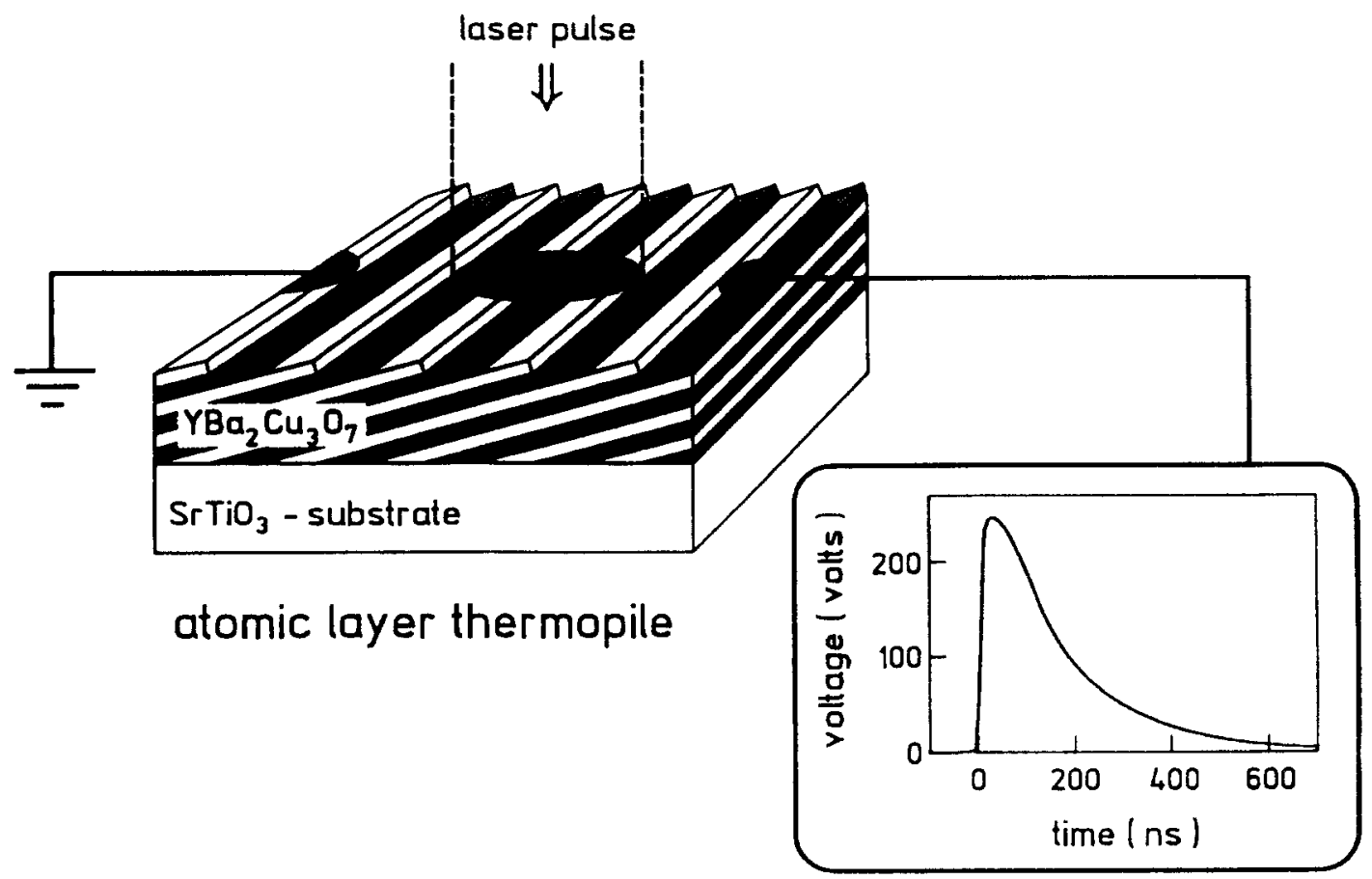

Figure 1. Atomic layer thermopile

$E_{c}=S_{c}\left(T_{2}-T_{1}\right) / d$

where $S_{c}$ is the Seebeck coefficient for the cdirection of $\mathrm{YBCO}, T_{2}-T_{1}$ is the temperature difference and $d$ the sample thickness. If we apply a temperature gradient along the $a b-$ plane, then the thermoelectric field which is created within the sample is characterized by a Seebeck coefficient $S_{a b}$. It turns out that by doping the film $S_{a b}$ can be smaller than $S_{c}$. $S_{a b}$ depends strongly on the oxygen concentration $\delta$ and can be negative or positive; for further discussion we assume $S_{a b}=0$. If we now have a film with the c-axis at an angle $\alpha$ with respect to the film surface, then a temperature gradient perpendicular to the film surface is effective only along the c-direction and we will have a thermoelectric field along the c-direction which is proportional to the component of the temperature gradient along the c-direction, $\nabla T \cos \alpha$.

This electric field $E_{c}$ now has components, a longitudinal component $E_{\text {long }}$ which is parallel to the temperature gradient and a transverse component which is transverse to the temperature gradient. The transverse component has a value.

$E_{\text {trans }}=S_{c} \nabla T \sin \alpha \cos \alpha$

Accordingly, we obtain a transverse voltage $U_{\text {trans }}$ along the film surface

$U_{\text {trans }}=l / d\left(T_{2}-T_{1}\right) S_{\mathrm{c}} \cos \alpha \sin \alpha$

where $l$ is the separation of the electrical contacts. In case that $S_{a b}$ is not zero, $S_{c}$ in the last equation has to be replaced by $S_{c}-S_{a b}$. For $l=1 \mathrm{~cm}$ and assuming a large temperature difference that can be generated by laser irradiation, namely of $300 \mathrm{~K}$ over $10^{-5} \mathrm{~cm}$, 


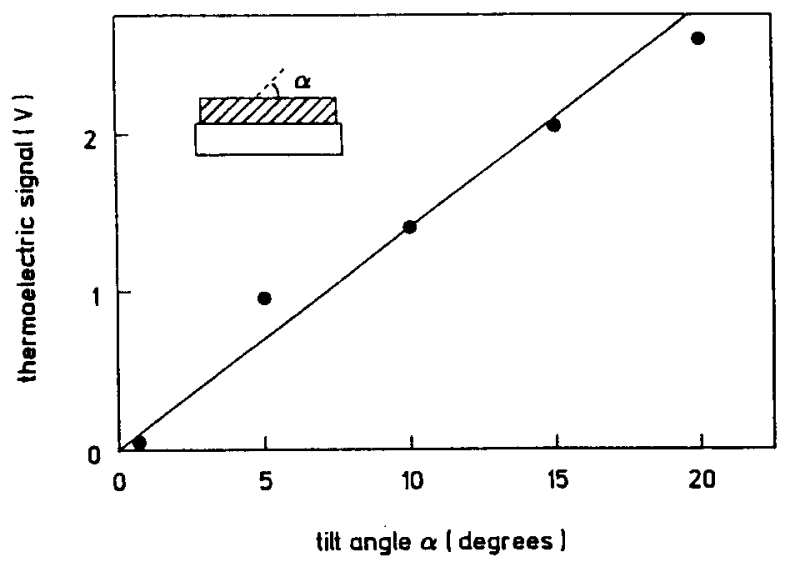

Figure 2. Thermoelectric signals for $\mathrm{YBCO}$ films of different tilt angles [7].

a difference of the Seebeck coefficient of $10^{-5}$ $\mathrm{V} / \mathrm{K}$ and $\cos \alpha \sin \alpha=1 / 3$ we find that the transverse voltage can reach a value of $\sim 100$ V.

The transverse voltage is proportional for small $\alpha$ directly to $\alpha$. This is indeed found experimentally (Fig. 2). Thermoelectric signals for different films of different tilt angles show, that the thermoelectric voltage increases almost linearly with angle $\alpha$, up to a tilt angle of $20^{\circ}$. The experiment clearly shows that the transverse thermoelectric voltage due to the transverse Seebeck effect depends on the geometric arrangement of the YBCO films relative to the film surface.

There are two aspects of these results. A small thermoelectric signal indicates a good orientation of the $c$-axis perpendicular to the film plane. For larger tilt angles the film is suitable as detector for optical radiation.

We have studied the response of the detector for different film thicknesses. The film thickness determines the heat diffusion from the film to the substrate. For small thickness the heat escapes fast, for thicker films the heat takes longer time to escape from the film. We have observed (Fig. 3) laser pulses of a $\mathrm{CO}_{2}$-laser with short spikes due to mode locking. For a $40 \mathrm{~nm}$ thick film the spikes are well resolved. The heat escapes in a few ns from the film into the substrate. The response time is few ns. For a film of larger thickness the thermovoltaic signal does no longer resolve the single laser pulses since the heat escapes slower from the film and accordingly, the time constant is larger. For a $300 \mathrm{~nm}$ thick film the time constant is about $100 \mathrm{~ns}$.

\section{Characteristic detector data}

We have found that the detector is suitable to detect radiation in a very large wavelength range. It responds to UV radiation, to visible radiation, to infrared radiation at $10 \mu \mathrm{m}$ and to far infrared radiation and even to radiation at millimeter waves. The detector can be used for detection of pulsed or $\mathrm{cw}$ radiation.

The signal increases almost linearly with laser power. A dynamical range that extends over more than 10 orders of magnitude has been found [7]. So the detector has an extremely large dynamical range.

We have built an array with strips, each with an electric connection for signal recording. Irradiation of the strips with laser radiation results in different signals. By this way we can determine profiles of laser radiation in one direction. By moving a slit in the other direction we have determined the profile of laser radiation in the plane of the beam. This method allows to adjust e.g. $\mathrm{cw}-\mathrm{CO}_{2}$ lasers or other lasers. The method represents a fast and simple way of laser controlling. 


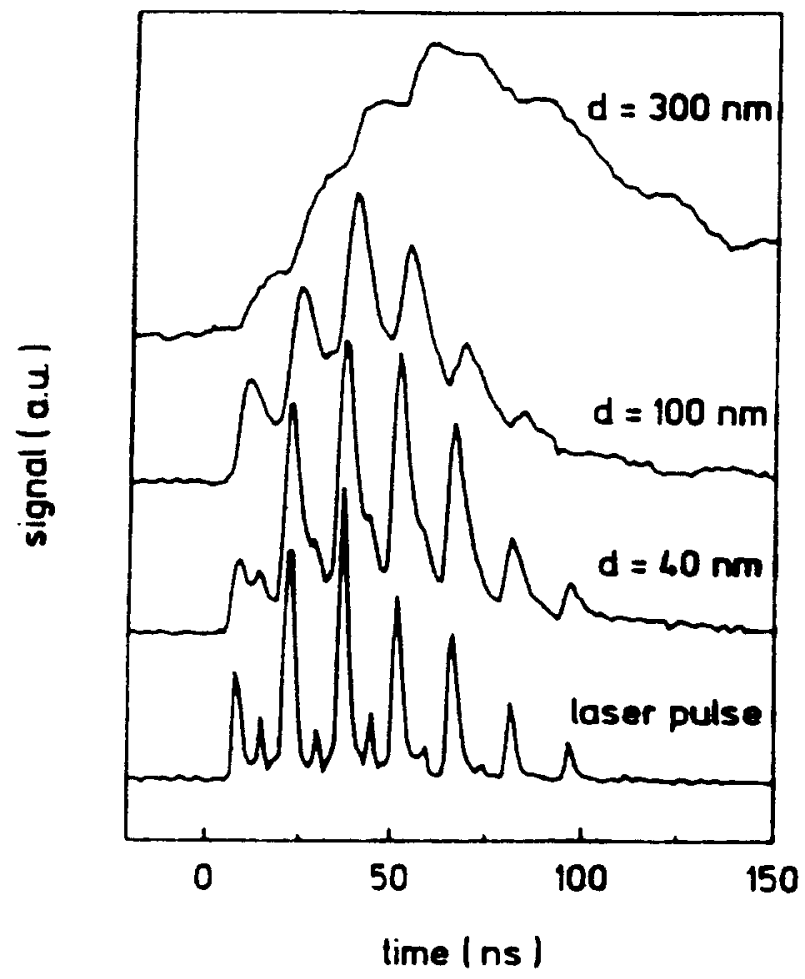

Figure 3. Response time of atomic layer thermopile [9]

\section{Summary}

Off-axis grown epitaxial YBCO films show a transverse Seebeck effect that is due to the anisotropy of YBCO. We have demonstrated the application of the effect for developing an optical radiation detector. The detector has a responsivity of about $1 \mathrm{mV} / \mathrm{W}$, a response time depending on film thickness between 1 ns and $100 \mathrm{~ns}$. The detector has an extremely large dynamical range. It responds to radiation over a very large wavelength range. It is suitable to detect millimeter wave radiation, infrared radiation, visible light and ultravi- olet radiation. In the ultraviolet it is especially suitable to detect excimer laser radiation. The detector can be applied for laser power measurements and also for laser beam profiling. The main application may be in contolling of lasers in material processing and medicine.

The work was supported by the Bayerische Forschungsstiftung through the Bayerischer Forschungsverbund Hochtemperatur-Supraleiter (FORSUPRA).

\section{References}

[1] C.L. Chang, A. Kleinhammer, W. G. Moulton, and L.R. Testardi, Phys. Rev. B 41, 11564 (1990).

[2] K.L. Tate, R.D. Johnson, C.L. Chang, E.F. Hilinski, and S.C. Foster, J. Appl. Phys. 67, 4375 (1990).

[3] H.S. Kwok and J.P. Zheng, Phys. Rev. B 46, 3692 (1992).

[4] H. Lengfellner, G. Kremb, A. Schnellbögl, J. Betz, K.F. Renk and W. Prettl, Appl. Phys. Lett. 60, 501 (1992).

[5] J.F. Scott, Appl. Phys. Lett. 56, 1914 (1990).

[6] D. Mihailovic and A. Heeger, Solid State Commun. 75, 319 (1990).

[7] H. Lengfellner, S. Zeuner, W. Prettl and K.F. Renk, Europhys. Lett. 25, 375 (1994).

[8] L.R. Testardi, Appl. Phys. Lett. 64, 2347 (1994).

[9] P.G. Huggard, S. Zeuner, K. Goller, H. Lengfellner and W. Prettl, J. Appl. Phys. 75, 616 (1994). 